\title{
Congenital Vertebral Malformations In French Bulldogs: X-Ray Vs Computed Tomography
}

\author{
Corlat L ${ }^{1 *}$, Blanco $\mathrm{B}^{2}$, Lucena $\mathrm{R}^{2}$, Ginel $\mathrm{PJ}^{2}$, Miró $\mathrm{F}^{3}$, Novales $\mathrm{M}^{2}$ \\ ${ }^{1}$ Dep. of Semiology and Medical Pathology, Faculty of Veterinary Medicine, USAMV, Iasi, Romania \\ ${ }^{2}$ Dep. of Animal Medicine and Surgery, Faculty of Veterinary Medicine, University of Cordoba, Spain \\ ${ }^{3}$ Dep. of Anatomy and Comparative Pathology, Faculty of Veterinary Medicine, University of Cordoba, \\ Spain \\ * corresponding author: lucia_corlat@yahoo.ro
}

Bulletin UASVM Veterinary Medicine 74(1) / 2017,

Print ISSN 1843-5270; Electronic ISSN 1843-5378

DOI:10.15835/buasvmcn-vm: 11508

\begin{abstract}
Congenital vertebral malformations of the thoracolumbar area can have an important impact in the clinical evolution of French Bulldogs due to the instability it creates at the spinal level. The aim of this study is to show the differences between x-ray and CT scans in vertebral malformations of the French Bulldog. CT scans can offer a higher degree of certainty in the diagnosis of congenital vertebral malformations of the dogs. The VR model can offer a more thorough evaluation of the existing modifications of the vertebral body, allowing the examiner to circumvent the superposition effect than can be observed in the x-ray views and offering the chance to evaluate whether there is scoliosis or kyphosis present.
\end{abstract}

Keywords: Computed tomography, dog, vertebral malformations, $x$-ray, section, incidence

\section{INTRODUCTION}

Congenital vertebral malformations of the thoracolumbar area can have an important impact in the clinical evolution of French Bulldogs due to the instability they induce at the spinal level, leading to local inflammations, chronic lumbar pain, lameness and neurological disorders [Humbert et al., 2012; Moissonnier, 2011).

The main types of congenital vertebral malformation have been established using the Nasca classification grid [Lappalainen et al., 2012; Gutierrez-Quintana et al., 2014; Besalti et al., 2005)

\section{AIMS}

The aim of this study was to show the differences between x-ray and CT scan in vertebral malformations of the French Bulldog.

\section{MATERIALS AND METHODS}

Twenty-one French Bulldogs (13 female, 8 males) with an age range 1 to 11 years old were included in the study. All dogs showed clinical signs compatible with medullar compression syndrome (lumbar pain, lameness in hind limbs or in one of the anterior limbs, urine and faecal retention). CT scans of the thoracolumbar spine area were taken for 13 of these dogs ( 4 males and 9 females), whereas for the other 8 dogs ( 4 males and 4 females) thoracolumbar spine x-rays were taken in orthogonal recumbences (lateral and ventrodorsal).

The radiologic study was performed on sedated dogs in lateral and ventrodorsal views. The exposure parameters were $65 \mathrm{kVp}$ and 2.4 mAs. The study was performed with a stationary X-ray machine (Odel model). Radiographs were processed by computerized radiology (Fuji CR). The CT scans were taken with the patients under general anaesthesia placed in a dorsal decubitus, 


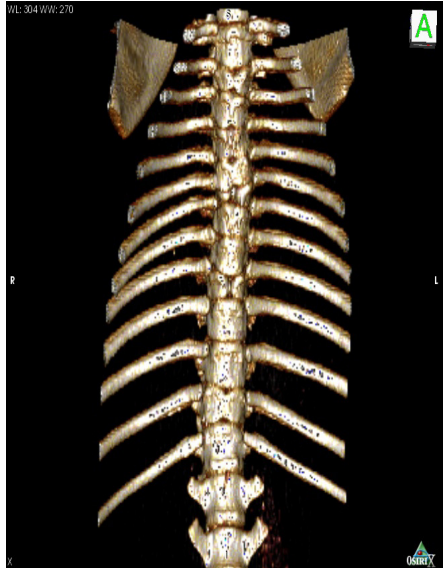

Fig. 1. A VR reconstruction of a CT scan of the thoracic spine of a female French Bulldog, 5 years. Multiple malformed vertebrae - butterfly (T6, T9), ventral wedge (T2, T4, T12) and shortened types (T7, T8, T10).

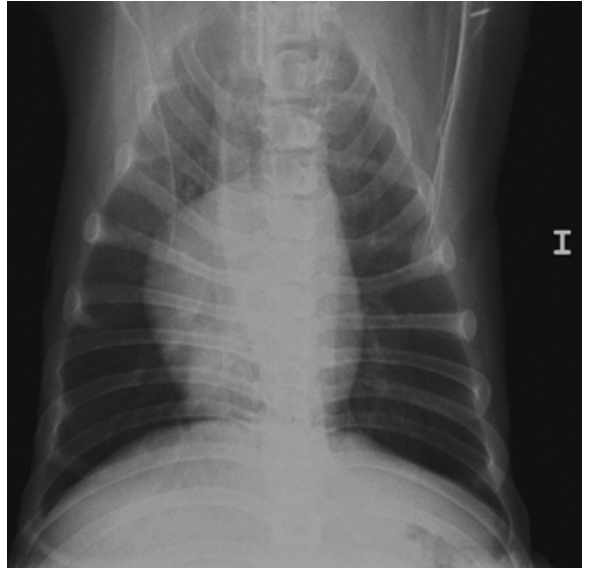

Fig. 2. X-ray image of the same patient, female French Bulldog, in a dorsal recumbence. Note the superposition effect of the thoracic vertebrae with the cardiac silhouette. The malformed vertebrae can be suspected noting the modified angles of the ribs.

\section{Number of vertebrae}

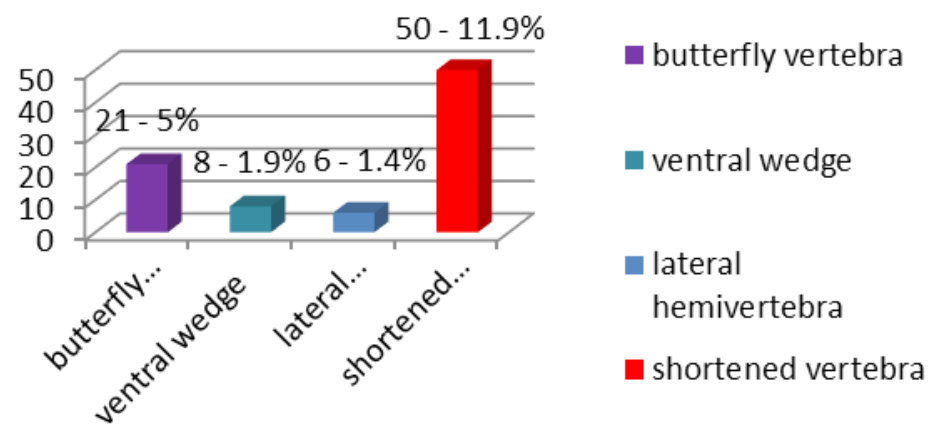

Fig. 1. Value distribution of the most encountered types of vertebral malformations in the 21 dogs included in the study.

using a High Speed Dual (General Electric) machine. The exposure parameters were $120 \mathrm{kV}$, $40 \mathrm{~mA}$, using algorithms for bone and soft tissue. Sections of $0,6 \mathrm{~mm}$ were made, in helical mode with a pitch of 0.75 . The images had been examined in transverse (axial) incidence and, in reformatted images, in dorsal and sagittal planes. The selected area for scanning has been reconstructed in a 3D image using the volume rendering (VR) technique.

The butterfly vertebra was the most encountered vertebral malformation, followed by ventral hypoplasia of the vertebral body (ventral wedge shape) and shortened vertebrae.

\section{RESULTS}

X-ray represented a good diagnostic tool to detect vertebral malformation in lateral views but the diagnosis might be difficult in ventrodorsal surveys especially if alterations are located in the thoracic vertebrae as the spinal processes and the sternum could hide the vertebral body. The CT scan showed good details in the three planes (axial, sagittal, dorsal planes) and thus represented a better chance for evaluating the impact of the malformed vertebral bodies on the spinal cord - compressive lesions, disc hernias. The VR model permitted a more throughout evaluation of the existing modifications of the vertebral body, avoiding the superposition effect than could be observed in the x-ray views and allowing to evaluate the impact of the vertebral body malformation on the general line of the spine - whether scoliosis or kyphosis are present.

The most frequent vertebral malformations have been: butterfly vertebra (21 vertebrae, 15 
dogs - 5\%), ventral wedge (8 vertebrae, 6 dogs $1.9 \%$ ), lateral hemivertebra (6 vertebrae, 4 dogs $1.4 \%$ ) and shortened vertebrae (50 vertebrae, 18 dogs - 11.9\%), out of 420 vertebrae evaluated (21 dogs). The number of vertebrae affected in one dog ranged from 0 to 9 , with a mean number of 5 .

\section{CONCLUSION}

CT scan represents a more sensible tool for the diagnosis of congenital vertebral malformations of dogs. The VR reconstruction of the thoracic section of the spine can circumvent the superposition phenomenon found with radiographic surveys that could worsen the evaluation of the vertebral body modifications.

\section{REFERENCES}

1. Humbert L, Steffen JS, Vialle R, Dubousset J, Vital JM, Skalli W (2012). 3D analysis of congenital scoliosis due to hemivertebra using biplanar radiography. Eur Spine J 22:379-386.

2. Lappalainen AK, Salomaa R, Junnila J, Snellman M, Laitinen-Vapaavuori O (2012). Alternative classification and screening protocol for transitional lumbosacral vertebra in German shepherd dogs. Acta Veterinaria Scandinavica 54:27.

3. Gutierrez-Quintana R, Guevar J, et al. (2014). A proposed radiographic classification theme for congenital thoracic vertebral malformations in brachycephalic "screw-tailed" dog breeds. Vet Radiol Ultrasound 55(6):585-591.

4. Moissonnier P (2011). Thoracic kyphosis associated with hemivertebra. Veterinary Surgery 40:1029-1032.

5. Besalti O, Ozak A, Pekcan Z, Eminaga S (2005). Nasca classification of hemivertebra in five dogs. Irish Veterinary Journal Volume 58: 688-690. 